This is an author-created, un-copyedited version of an article accepted for publication in Physical Review Letters. The publisher is not responsible for any errors or omissions in this version of the manuscript or any version derived from it. The Version of Record is available online at http://dx.doi.org/10.1103/PhysRevLett.116.106802.

Robust Phonon-Plasmon Coupling in Quasifreestanding Graphene on Silicon Carbide R. J. Koch, S. Fryska, M. Ostler, M. Endlich, F. Speck, T. Hänsel, J. A. Schaefer, and Th. Seyller Physical Review Letters 116 (2016) 106802.

(c) 2016 American Physical Society 


\title{
Robust phonon-plasmon coupling in quasi-freestanding graphene on silicon carbide
}

\author{
R. J. Koch, ${ }^{1,2,3,4, *}$ S. Fryska, ${ }^{2}$ M. Ostler, ${ }^{1,2}$ M. Endlich, ${ }^{3}$ F. \\ Speck, ${ }^{1,2}$ T. Hänsel, ${ }^{3,4}$ J. A. Schaefer, ${ }^{3,4,5}$ and Th. Seyller ${ }^{1,2}$ \\ ${ }^{1}$ Institut für Physik, Technische Universität Chemnitz, Germany \\ ${ }^{2}$ Lehrstuhl für Technische Physik, Universität Erlangen-Nürnberg, Germany \\ ${ }^{3}$ Institut für Physik, Technische Universität Ilmenau, Germany \\ ${ }^{4}$ Institut für Mikro- und Nanotechnologien, Technische Universität Ilmenau, Germany \\ ${ }^{5}$ Department of Physics, Montana State University, USA
}

(Dated: April 26, 2018)

\begin{abstract}
Using inelastic electron scattering in combination with dielectric theory simulations on differently prepared graphene layers on silicon carbide we demonstrate that the coupling between the 2D plasmon of graphene and the surface optical phonon of the substrate cannot be quenched by modification of the interface via intercalation. The intercalation rather provides additional modes like, e.g., the silicon-hydrogen stretch mode in the case of hydrogen intercalation or the silicon-oxygen vibrations for water intercalation that couple to the $2 \mathrm{D}$ plasmons of graphene. Furthermore, in the case of bilayer graphene with broken inversion symmetry due charge imbalance between the layers, we observe a similar coupling of the $2 \mathrm{D}$ plasmon to an internal infrared-active mode, the LO phonon mode. The coupling of graphene plasmons to vibrational modes of the substrate surface and internal infrared active modes is envisioned to provide an excellent tool for tayloring the plasmon band structure of monolayer and bilayer graphene for plasmonic devices such as plasmon filters or plasmonic wave guides. The rigidity of the effect furthermore suggest that it may be of importance for other $2 \mathrm{D}$ materials as well.
\end{abstract}

PACS numbers: 12.34

Graphene is a two-dimensional crystal which has gained considerable attention since its unusual transport properties were reported for the first time [1-3]. In particular, epitaxial graphene (EG) on silicon carbide ( $\mathrm{SiC})$ is of great interest due to its potential applications in electronic devices $[4,5]$. SiC is already used in electronic devices and $\mathrm{EG}$ on $\mathrm{SiC}$ has been studied for several years now [6]. The growth of EG has been perfected towards wafer scale homogeneous films [7, 8]. Still, many of the interactions between graphene and the $\mathrm{SiC}$ substrate have to be understood. For example, the charge carrier dynamics may be influenced by the long-range coupling to polar modes [9]. The resulting remote scattering is important for future graphene devices, especially plasmonic devices, because the plasmon dispersion itself is strongly influenced [10-12].

$\mathrm{EG}$ on $\mathrm{SiC}$ exists in different varieties. On the Siterminated $\mathrm{SiC}(0001)$ surface, graphene resides on the so-called buffer layer which itself constitutes a honeycomb arrangement of carbon atoms. However, the buffer layer shows no graphene-like dispersion of the $\pi$-band due to hybridization with the substrate [13]. Recently, several groups demonstrated that the buffer layer can be converted into so-called quasi-freestanding graphene by intercalating different elements underneath [14]. The alteration of the interface beween graphene and $\mathrm{SiC}$ is expected to lead to different couplings of the charge carrier plasmons with vibronic excitations of the substrate.

In the present work, we use high (energy-)resolution electron energy loss spectroscopy (HREELS) in combination with simulations based on dielectric theory to study this coupling for three different cases: epitaxial graphene on the buffer layer (MLG), quasi-freestanding monolayer graphene on hydrogen terminated $\mathrm{SiC}(0001)$ (H-QFMLG) and on an oxygen terminated $\mathrm{SiC}(0001)$ surface (O-QFMLG). Furthermore, we demonstrate that an equivalent coupling to an internal mode exists for the case of bilayer graphene with broken inversion symmetry. Here, the plasmons couple to the optical phonon mode of the graphene bilayer.

Graphene was grown on semi-insulating $\mathrm{SiC}$ by sublimation growth as described elsewhere [7] using a custom built setup [15]. H-QFMLG was obtained by annealing the buffer layer in molecular hydrogen $[16,17]$, while OQFMLG was prepared by annealing in water vapor [18]. Quasi-freestanding bilayer graphene (H-QFBLG) on $\mathrm{H}$ terminated $\mathrm{SiC}(0001)$ was prepared by annealing MLG in hydrogen $[16,19]$. Details are provided in the Supplemental Material [20].

HREELS is a powerful tool to investigate surface [21] and, as we will show, also subsurface adsorbates. It furthermore provides an experimental access to surface phonon and plasmon modes on a wide variety of materials [22]. The momentum transfer is calculated using the relation

$$
q_{\|}=\frac{\sqrt{2 m_{e}}}{\hbar}\left[\sqrt{E} \sin \left(\theta_{0}\right)-\sqrt{E-\hbar \omega} \sin \left(\theta_{s}\right)\right],
$$

where $\theta_{0}$ is the angle of incidence of the electrons with energy $E, \theta_{s}$ the scattering angle, and $\hbar \omega$ the energy loss suffered by the inelastically scattered electrons. Hence, the dispersion of the mode can be mapped by scanning 


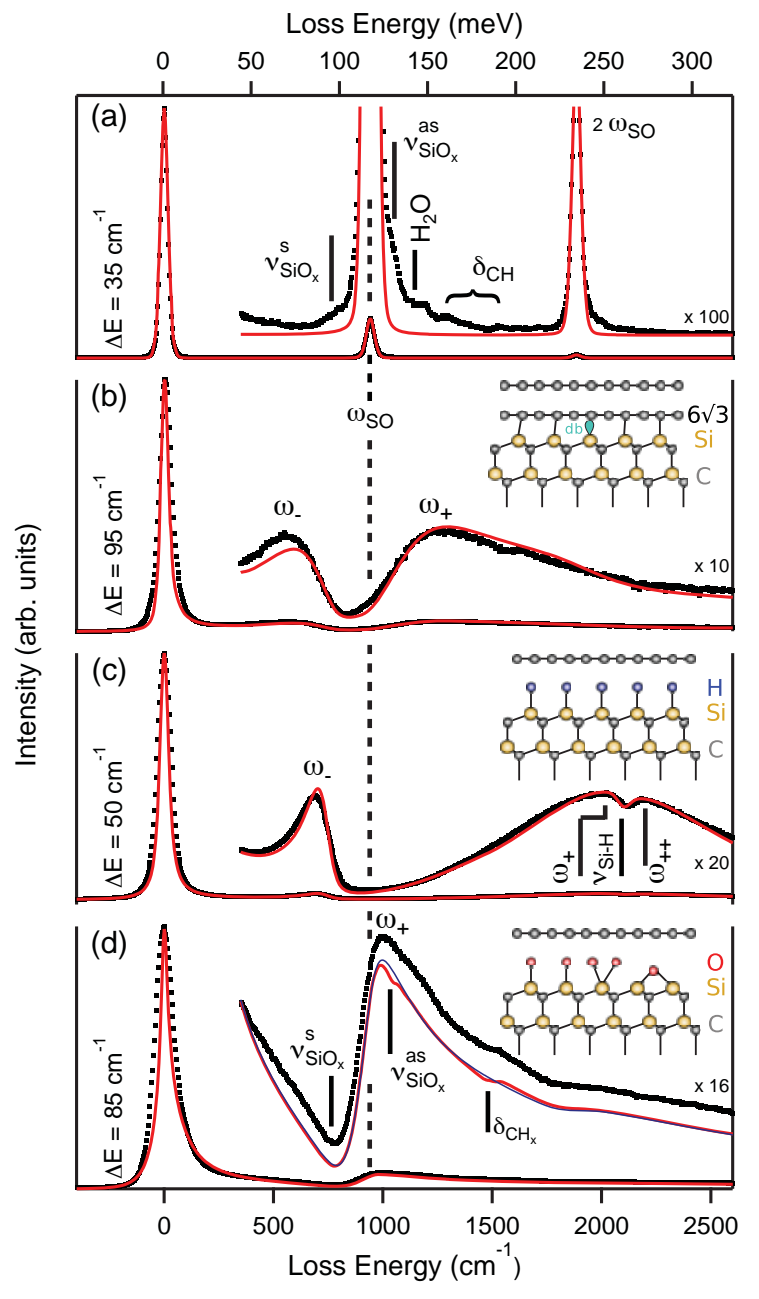

FIG. 1. HREELS spectra (black dots) and dielectric theory simulations (red line) of bare hydrogen etched silicon carbide (a), MLG (b) H-QFMLG (c) and O-QFMLG (d). The primary beam energy was set to $E=37 \mathrm{eV}$ for (a), $E=21 \mathrm{eV}$ for (b,d) and $E=11 \mathrm{eV}$ for (c). (a) was taken at room temperature, (b-d) were taken at $T=130 \mathrm{~K}$. The simulated curves in (d) are offset from the measured curve for clarity.

$\theta_{s}$ while keeping all other parameters constant. This socalled off-specular scattering geometry is frequently used to determine surface phonon dispersions, but it was also employed previously to study graphene plasmons [23-25]. Here we use the so-called specular scattering geometry, where $\theta_{s}=\theta_{0}$ and the primary energy $E$ is varied. This measurement geometry allows us to facilitate the interpretation, by simulating the HREELS spectra using dielectric theory as explained in Refs. 26-28.

Fig. 1 shows HREELS spectra of bare, hydrogen etched silicon carbide (a), MLG (b), H-QFMLG (c), and O-QFMLG (d). The spectrum of the bare SiC surface in fig. 1(a) shows an intense loss signal from the surface optical (SO) phonon of $\mathrm{SiC}\left(\omega_{\mathrm{SO}}=940 \mathrm{~cm}^{-1}\right)$ and its double loss signal $\left(2 \times \omega_{\mathrm{SO}}=1880 \mathrm{~cm}^{-1}\right)$. In addition,
TABLE I. Parameters used for dielectric theory simulations shown in fig. $1 . d$ is the thickness of the graphene stack. $\omega_{P L}$ is the dispersion independent plasmon frequency with $\omega_{P 2 D} \sim \omega_{P L} \sqrt{q_{\|} d}[26]$.

\begin{tabular}{l|ccc|ccc} 
Sample & $\frac{d}{\mathrm{~nm}}$ & $\frac{\omega_{\mathrm{Pl}}}{\mathrm{cm}^{-1}}$ & $\frac{\Gamma}{\mathrm{cm}^{-1}}$ & $\frac{n}{10^{12} \mathrm{~cm}^{-2}}$ & $\frac{m^{*}}{m_{e}}$ & $\frac{\mu}{\mathrm{cm}^{2} / \mathrm{Vs}^{2}}$ \\
\hline MLG & 0.5 & 14500 & 145 & $10.0[7]$ & 0.017 & 3780 \\
H-QFMLG & 0.35 & 20200 & 123 & $6.0[17]$ & 0.008 & 10100 \\
O-QFMLG & 0.35 & 16900 & 2366 & $8.0[18]$ & 0.014 & 280
\end{tabular}

several other features are visible which are due to water, $\mathrm{CH}$ groups, and $\mathrm{SiO}$ groups. The reason for these features is that the hydrogen etched surface is partially covered by oxides and atmospheric contaminations which have not been removed completely. They are, however, of no importance for the following discussion. The red line in fig. 1(a) is the result of a simulation of the spectrum [26] which takes into account the SO phonon and the charge carrier density in the $\mathrm{SiC}$ bulk. The latter gives rise to the broadening of the spectrum. The simulation parameters obtained in this way for the $\mathrm{SiC}$ bulk were used for the simulations of the spectra obtained from the graphene/SiC stack discussed in the following. The spectrum of MLG is shown in fig. 1(b). In contrast to the bare $\mathrm{SiC}$ surface no contaminations are visible. Notably, the $\mathrm{SO}$ phonon of the $\mathrm{SiC}$ substrate is quenched and two new phonon-plasmon coupled modes $\omega_{-}$and $\omega_{+}$appear as described earlier [26]. The changes in the spectrum are well captured by the dielectric theory simulations which now take into account the $\mathrm{SiC}$ bulk and a graphene layer.

A similar behavior is observed for the other two samples. The spectrum of H-QFMLG shown in fig. 1(c) is again characterized by the two phonon-plasmon coupled modes $\omega_{-}$and $\omega_{+}$with energetic positions which will be discussed further below. This observation indicates that the observed coupling between the SO phonon of $\mathrm{SiC}$ and the graphene plasmons is very robust. Moreover, it suggests that the coupling of the plasmons to optical phonons of any substrate, e.g. $\mathrm{SiO}_{2}$ [29], cannot be lifted by interface modifications. Instead, we observe that additional vibrational degrees of freedom located at the interface couple to the graphene plasmons as well. This is seen from the appearance of an additional feature in the spectrum in fig. 1(c) at $2090 \mathrm{~cm}^{-1}$. Here, a coupling between the $\mathrm{Si}-\mathrm{H}$ stretch mode of the H-saturated $\mathrm{SiC}(0001)$ surface and the $\omega_{+}$plasmon occurs, leading to a dip in the spectrum, due to the avoided crossing in the coupled phonon-plasmon dispersion as will be shown later, as well as an additional maximum labeled $\omega_{++}$. The dielectric theory simulation (red curve), which included a Si-H stretch mode of $\nu_{\mathrm{SiH}}=2090 \mathrm{~cm}^{-1}$ describes the measured spectrum very well. This shows that HREELS is capable of detecting subsurface interface vibrations.

In the case of O-QFMLG an equivalent behavior is ob- 
served [see fig. 1(d)]. Here, features related to the symmetric $\mathrm{SiO}_{x}$ stretch vibration $\left(\nu_{\mathrm{SiO}_{x}}^{\mathrm{s}}=780 \mathrm{~cm}^{-1}\right)$ and to the antisymmetric $\mathrm{SiO}_{x}$ stretch vibration $\left(\nu_{\mathrm{SiO}_{x}}^{\mathrm{as}}=\right.$ $1050 \mathrm{~cm}^{-1}$ ) can be identified. Furthermore, an additional feature is observed at around $1480 \mathrm{~cm}^{-1}$, which we tentatively assign to the $\mathrm{C}-\mathrm{H}$ bending mode $\delta_{\mathrm{CH}_{x}}$. Although these modes give rise to very subtle changes in the spectrum, their presence is clearly demonstrated by comparing the measured spectra with the simulated ones. As an example we show in fig. 1(d) two different simulations which were carried out with (red curve) and without (blue curve) including these additional modes. While the presence of the $\mathrm{SiO}_{x}$ related features is expected [18], the presence of C-H bonds has not been observed before. Considering the large density of defects observed by Raman spectroscopy [18] the presence of C$\mathrm{H}$ bonds in quantities below the detection limit of $\mathrm{x}$-ray photoelectron spectroscopy is reasonable.

The simulations of the HREELS spectra enables us to estimate the charge carrier mobility $\mu$ and the effective mass $m^{*}$ by using the relations $\omega_{\mathrm{Pl}}=\sqrt{n e^{2} / \epsilon m^{*}}$ and $\Gamma=e / \mu m^{*}$, where $n, \omega_{\mathrm{Pl}}$, and $\Gamma$ are the charge carrier density, plasmon frequency, and plasmon damping, respectively. The static dielectric constant $\epsilon$ was estimated by the average of the dielectric constants of the SiC substrate and vacuum resulting in a value of 5 . $n$ was taken from the literature $[7,17,18]$, where it was gathered from Hall effect measurements. $\omega_{\mathrm{Pl}}$ and $\Gamma$ were adjusted to optimize the agreement between the measured and the calculated loss spectra. The resulting charge carrier mobility and effective mass values are compiled in table I together with the other parameters. The effective masses are about one order of magnitude lower than the theoretical calculations in the long wavelength limit by Abedinpour et al. [30] suggest. However, compared to optical measurements [31], HREELS measures at a rather large momentum transfer thus suggesting a momentum dispersion of the effective mass. The mobility values show reasonable agreement with values determined by transport measurements $[7,17,18]$. Note that transport properties are determined by the long wave length limit of the effective mass. Hence, the charge carrier mobility estimated from the fits of the HREELS spectra may deviate from the mobility determined by Hall effect experiments. Nevertheless, the numerical values determined by the two methods are of the same order of magnitude.

Next, we determined the dispersion of the maxima in the energy loss spectra of fig. 1 and spectra obtained for other primary beam energies in the range between $2 \mathrm{eV}$ and $80 \mathrm{eV}$. The dispersion relations of the coupled phonon-plasmon modes are shown as black circles in fig. 2. In addition, the surface loss function $[20,22]$ is plotted there as a color coded intensity map. The dielectric function $\epsilon\left(\omega, q_{\|}\right)$was obtained with a formalism developed by Lambin et al. [27] applied to a three layer model including graphene, the interface, and an infinitely thick silicon
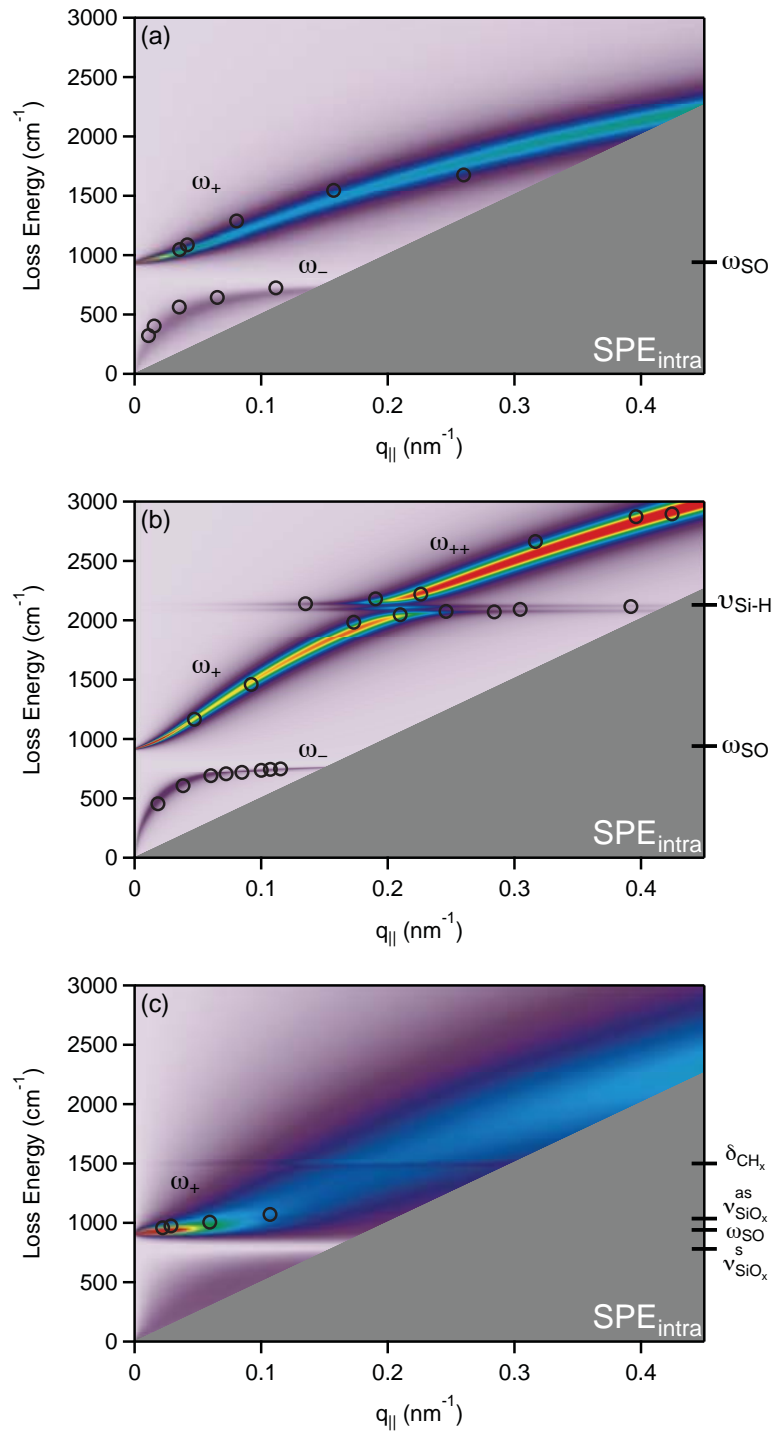

FIG. 2. Dispersion of the phonon-plasmon coupled modes of (a) MLG, (b) H-QFMLG, and (c) O-QFMLG. The calculated surface loss function is shown as a $2 \mathrm{D}$ color coded intensity map. The three branches of the coupled phonon plasmon dispersion are marked by $\omega_{-}, \omega_{+}$and $\omega_{++}$. Black circles mark peak positions in the HREELS spectra taken at different primary beam energies. For water intercalated graphene only the $\omega_{+}$branch was resolved well enough to be included in the plot. The gray shaded triangle corresponds to the area where single particle electron hole excitations are possible $\left(\mathrm{SPE}_{\text {intra }}\right)$.

carbide layer representing the substrate. For details see the supplementary information. In the case of MLG no interface was included as the buffer layer apparently does not lead to additional coupled modes. The gray region marks the regime of single particle intraband excitations $\left(\mathrm{SPE}_{\text {intra }}\right)$, in which the plasmon mode would be strongly damped [32].

The dispersion of MLG displayed in fig. 2(a) shows the 
expected behavior [26]. The plasmon couples to the SO phonon of the $\mathrm{SiC}$ substrate leading to the two modes $\omega_{-}$and $\omega_{+}$with an avoided crossing in between. The measured dispersion agrees very well with the simulation of the loss function. In comparison to ref. 26 , where the analytical expression used to calculate the dispersion neglected damping, the inclusion of the damping here leads to an improved agreement between experiment and theory. H-QFMLG [see fig. 2(b)] shows a dispersion very close to the one of a conventional 2DEG with the exception of the two avoided crossings. The first one, which is also present in MLG, originates from the coupling of the plasmon to the $\mathrm{SO}$ phonon of the $\mathrm{SiC}$ substrate. Here $\omega_{-}$converges to $\omega_{T O}$ of $\mathrm{SiC}$ for high momentum transfers and $\omega_{+}$to $\omega_{S O}$ of SiC for $q_{\|} \rightarrow 0$. The second gap is caused by the coupling of the plasmon to the $\mathrm{Si}-\mathrm{H}$ stretching vibration at the graphene/SiC interface. Consequently, $\omega_{+}$converges to $2090 \mathrm{~cm}^{-1}$ for high momentum transfers, while $\omega_{++}$converges to the Si-H stretching frequency of $2130 \mathrm{~cm}^{-1}$ for $q_{\|} \rightarrow 0$ which was previously observed by infrared absorption spectroscopy [19, 33, 34] where the momentum transfer is very small.

Now we turn to the water intercalated sample [fig. $2(\mathrm{c})]$. The coupling of the plasmon with the SO phonon of $\mathrm{SiC}$ is again apparent. However, we note that the lower lying mode $\omega_{-}$is only visible as a shoulder in the elastic peak [see fig. 1(d)]. This is due to a higher damping of the plasmon which is caused by the low charge carrier mobility of O-QFMLG as compared to MLG or H-QFMLG $[17,18]$ and which reflects itself in a strong broadening of the modes. The latter also applies to the higher lying mode $\omega_{+}$. In the simulated surface loss function, the consequence is visible in a strong broadening of the $\omega_{+}$dispersion branch and the absence of a clear maximum in the $\omega_{-}$dispersion branch, in agreement with the HREELS measurements. The high damping also has consequences for the dispersion. First, an increase of the damping results in a reduced dispersion, which is obvious when comparing the data of the three different samples: the sample with the highest charge carrier mobility (lowest damping) exhibits the strongest dispersion in both the experimental data as well as in the simulation. In addition, but not quite as obvious, an increase of the damping results in a stronger deviation of the dispersion from the $\sqrt{q}$-behavior of an undamped 2DEG plasmon. The simulation of the surface loss function of O-QFMLG included interface vibrations as discussed above. They manifest themselves as thin horizontal lines in the loss function. In agreement with that, they are only visible as weak shoulders in the experiment [see fig. 1(d)].

Finally we discuss the case of bilayer graphene where the LO phonon is normally not dipole active. However, breaking the inversion symmetry of AB-stacked bilayer graphene, for example by a charge imbalance between the two layers, a dipole active LO mode can be induced $[35,36]$. In our experiment we used quasi-freestanding bi-

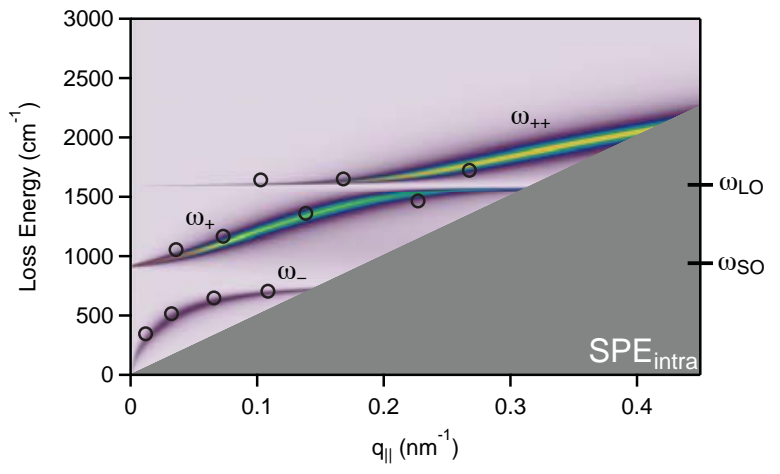

FIG. 3. Calculated surface loss function as a 2D color coded intensity map for H-QFBLG. The three branches of the coupled phonon plasmon dispersion are marked by $\omega_{-}, \omega_{+}$and $\omega_{++}$. Black circles mark the peak positions in the HREELS spectra taken at different primary beam energies. The gray shaded triangle corresponds to the area where single particle electron hole excitations are possible $\left(\mathrm{SPE}_{\text {intra }}\right)$.

layer graphene on $\mathrm{H}$-saturated $\mathrm{SiC}(0001)$ where a charge imbalance occurs spontaneously [37-39]. This leads, as for the substrate and interface dipole active modes, to an additional branch in the phonon plasmon dispersion. This is demonstrated in fig. 3 which shows the measured dispersion (black circles) as well as the simulated surface loss function $P\left(\omega, q_{\|}\right)$in which a single LO phonon at $\omega_{L O}=1600 \mathrm{~cm}^{-1}$ was included.

In summary we have shown that the phonon plasmon coupling between the graphene's free charge carrier plasmon and the surface optical phonon of the SiC substrate cannot be lifted by intercalation. In all studied cases of graphene on $\mathrm{SiC}$ which included graphene on the buffer layer and quasi-freestanding graphene obtained by hydrogen or water intercalation the splitting into $\omega_{-}$and $\omega_{+}$modes was observed. The additional dipole active modes at the interface introduced by intercalation such as, e.g., Si-H or Si-O modes were observed to cause an additional splitting with an avoided crossing in a similar fashion as the substrate SO mode. Furthermore, for bilayer graphene with broken inversion symmetry the LO phonon was observed to couple with the plasmon, too. The observations were well captured by dielectric theory simulations. The comparison between experimental HREELS data and simulated surface loss functions provided additional information about the influence of damping/charge carrier mobility on the dispersion and broadening of the plasmon modes. In general, a low charge carrier mobility (high damping) reduces the dispersion and broadens the plasmon modes.

The observed strong modifications of the graphene free charge carrier plasmon will have a tremendous impact on the application of graphene for future plasmonic devices. The observations suggest that proper choice of the substrate as well as design of the interface could be em- 
ployed to create gaps in the 2DEG plasmon dispersion of graphene and thus to taylor it for specific applications, for example as a plasmon filter or in plasmonic wave guides. The observation of the coupling of the $2 \mathrm{D}$ plasmon to the LO phonon mode in bilayer graphene with broken inversion symmetry is particular intriguing because the symmetry breaking and thus the plasmonic gap can be switched on and off by an external electric field $[35,36]$ provided by proper gates electrodes. Furthermore, based on the robustness of the observed coupling it can be expected that a similar plasmon phonon coupling will be present in other two-dimensional materials like, e.g., transition metal dichalcogenides or black phosphorus.

We gratefully acknowledge discussions with S.I.-U. Ahmed and technical assistance by G. Hartung and G. Schlosser. The research has received funding from the European Union Seventh Framework Programme under grant agreement ${ }^{\circ} 604391$ Graphene Flagship, from the DFG within the Collaborative Research Centre SFB 953 Synthetic Carbon Allotropes and within the Priority Programme SPP 1459 Graphene. M.E. acknowledges funding by the Carl-Zeiss foundation.

* roland.j.koch@gmail.com

[1] K. S. Novoselov, A. K. Geim, S. V. Morozov, D. Jiang, Y. Zhang, S. V. Dubonos, I. V. Grigorieva, and A. A. Firsov, Science 306,666 (2004).

[2] K. S. Novoselov, A. K. Geim, S. V. Morozov, D. Jiang, M. I. Katsnelson, I. V. Grigorieva, S. V. Dubonos, and A. A. Firsov, Nature (London) 438, 197 (2005).

[3] Y. Zhang, Y.-W. Tan, H. L. Stormer, and P. Kim, Nature (London) 438, 201 (2005)

[4] C. Berger, Z. Song, T. Li, X. Li, A. Y. Ogbazghi, R. Feng, Z. Dai, A. N. Marchenkov, E. H. Conrad, P. N. First, and W. A. de Heer, J. Phys. Chem. B 108, 19912 (2004).

[5] C. Berger, Z. Song, X. Li, X. Wu, N. Brown, C. Naud, D. Mayou, T. Li, J. Hass, A. N. Marchenkov, E. H. Conrad, P. N. First, and W. A. de Heer, Science 312, 1191 (2006).

[6] P. N. First, W. A. de Heer, T. Seyller, C. Berger, J. A. Stroscio, and J.-S. Moon, MRS Bull. 35, 296 (2010).

[7] K. V. Emtsev, A. Bostwick, K. Horn, J. Jobst, G. L. Kellogg, L. Ley, J. L. McChesney, T. Ohta, S. A. Reshanov, J. Roehrl, E. Rotenberg, A. K. Schmid, D. Waldmann, H. B. Weber, and T. Seyller, Nat. Mater. 8, 203 (2009).

[8] C. Virojanadara, M. Syv'ajarvi, R. Yakimova, L. I. Johansson, A. A. Zakharov, and T. Balasubramanian, Phys. Rev. B 78, 245403 (2008).

[9] S. Fratini and F. Guinea, Phys. Rev. B 77, 195415 (2008).

[10] X. Luo, T. Qiu, W. Lu, and Z. Ni, Mater. Sci. Eng. R 74, 351 (2013).

[11] T. Low and P. Avouris, ACS Nano 8, 1086 (2014).

[12] D. Basov, M. Fogler, A. Lanzara, F. Wang, and Y. Zhang, Rev. Mod. Phys. 86, 959 (2014).

[13] K. V. Emtsev, F. Speck, T. Seyller, L. Ley, and J. D. Riley, Phys. Rev. B 77, 155303 (2008).
[14] S. Forti and U. Starke, J. Phys. D 47, 094013 (2014).

[15] M. Ostler, F. Speck, M. Gick, and T. Seyller, Phys. Status Solidi B 247, 2924 (2010).

[16] C. Riedl, C. Coletti, T. Iwasaki, A. A. Zakharov, and U. Starke, Phys. Rev. Lett. 103, 246804 (2009).

[17] F. Speck, J. Jobst, F. Fromm, M. Ostler, D. Waldmann, M. Hundhausen, H. B. Weber, and T. Seyller, Appl. Phys. Lett. 99, 122106 (2011).

[18] M.Ostler, F. Fromm, R. J.Koch, P. Wehrfritz, F.Speck, H.Vita, S. Boettcher, K. Horn, and T. Seyller, Carbon 70, 258 (2014).

[19] F. Speck, M. Ostler, J. Roehrl, J. Jobst, D. Waldmann, M. Hundhausen, L. Ley, H. B. Weber, and T. Seyller, Mater. Sci. Forum 645648, 629 (2010).

[20] See Supplemental Material at http://link.aps.org/supplemental/10.1103/PhysRevLett.116.106802 for details on sample preparation and the dielectric theory simulations.

[21] F. S. Tautz and J. A. Schaefer, J. Appl. Phys. 84, 6636 (1998).

[22] H. Ibach and D. L. Mills, Electron Energy Loss Spectroscopy and Surface Vibrations (Academic Press, New York, 1982).

[23] Y. Liu, R. F. Willis, K. V. Emtsev, and T. Seyller, Phys. Rev. B 78, 201403 (2008).

[24] T. Langer, J. Baringhaus, H. Pfnuer, H. W. Schumacher, and C. Tegenkamp, New J. Phys. 12, 033017 (2010).

[25] T. Langer, H. Pfnuer, C. Tegenkamp, S. Forti, K. Emtsev, and U. Starke, New J. Phys. 14, 103045 (2012).

[26] R. J. Koch, T. Seyller, and J. A. Schaefer, Phys. Rev. B 82, 201413 (2010).

[27] P. Lambin, J. P. Vigneron, and A. A. Lucas, Phys. Rev. B 32, 8203 (1985).

[28] P. Lambin, J.-P. P. Vigneron, and A. A. Lucas, Comput. Phys. Commun. 60, 351 (1990).

[29] H. Yan, T. Low, W. Zhu, Y. Wu, M. Freitag, X. Li, F. Guinea, P. Avouris, and F. Xia, Nat. Photonics 7, 394 (2013).

[30] S. H. Abedinpour, G. Vignale, A. Principi, M. Polini, W. K. Tse, and A. H. Macdonald, Phys. Rev. B 84, 045429 (2011).

[31] I. Crassee, J. Levallois, A. L. Walter, M. Ostler, A. Bostwick, E. Rotenberg, T. Seyller, D. van der Marel, and A. B. Kuzmenko, Nat. Phys. 7, 48 (2011).

[32] A. Principi, G. Vignale, M. Carrega, and M. Polini, Phys. Rev. B 88, 195405 (2013).

[33] N. Sieber, B. F. Mantel, T. Seyller, J. Ristein, L. Ley, T. Heller, D. R. Batchelor, and D. Schmeisser, Appl. Phys. Lett. 78, 1216 (2001).

[34] H. Tsuchida, I. Kamata, and K. Izumi, Appl. Phys. Lett. 70, 3072 (1997)

[35] A. B. Kuzmenko, L. Benfatto, E. Cappelluti, I. Crassee, D. van der Marel, P. Blake, K. S. Novoselov, and A. K. Geim, Phys. Rev. Lett. 103, 116804 (2009).

[36] T.-T. Tang, Y. Zhang, C.-H. Park, B. Geng, C. Girit, Z. Hao, M. C. Martin, A. Zettl, M. F. Crommie, S. G. Louie, Y. R. Shen, and F. Wang, Nat. Nanotechnol. 5, $32(2010)$.

[37] T. Ohta, A. Bostwick, T. Seyller, K. Horn, and E. Rotenberg, Science 313, 951 (2006).

[38] S. Mammadov, J. Ristein, R. J. Koch, M. Ostler, C. Raidel, M. Wanke, R. Vasiliauskas, R. Yakimova, and T. Seyller, 2D Mater. 1, 035003 (2014).

[39] S. Ulstrup, J. C. Johannsen, F. Cilento, J. A. Miwa, 
A. Crepaldi, M. Zacchigna, C. Cacho, R. Chapman,

E. Springate, S. Mammadov, F. Fromm, C. Raidel, T.
Seyller, F. Parmigiani, M. Grioni, P. D. C. King, and P. Hofmann, Phys. Rev. Lett. 112, 257401 (2014). 


\title{
Supplementary Information: Robust phonon-plasmon coupling in quasi-freestanding graphene on silicon carbide
}

\author{
R. J. Koch, ${ }^{1,2,3,4}$ S. Fryska, ${ }^{2}$ M. Ostler,,${ }^{1,2}$ M. Endlich, ${ }^{3}$ F. \\ Speck, ${ }^{1,2}$ T. Hänsel, ${ }^{3,4}$ J. A. Schaefer, ${ }^{3,4,5}$ and Th. Seyller ${ }^{1,2}$ \\ ${ }^{1}$ Institut für Physik, Technische Universität Chemnitz, Germany \\ ${ }^{2}$ Lehrstuhl für Technische Physik, Universität Erlangen-Nürnberg, Germany \\ ${ }^{3}$ Institut für Physik, Technische Universität Ilmenau, Germany \\ ${ }^{4}$ Institut für Mikro- und Nanotechnologien, Technische Universität Ilmenau, Germany \\ ${ }^{5}$ Department of Physics, Montana State University, USA \\ (Dated: January 28, 2016)
}

\section{SAMPLE PREPARATION}

The samples were prepared on semi-insulating SiC purchased from II-IV Inc. Prior to the growth of graphene, the substrates were chemically cleaned in organic solvents (acetone, isopropanol and methanol) and a modified RCA process [1].

Monolayer graphene (MLG), i.e. graphene on top of the buffer layer, was obtained by sublimation growth in Argon [2] at a temperature of $1650^{\circ} \mathrm{C}$ in a custom built furnace described elsewhere [3]. The annealing time was 15 min and the Ar pressure and flow rate were set to 1 bar and $1 \mathrm{slm}$, respectively. In order to obtain quasi-freestanding monolayer samples, first a so-called buffer layer with $(6 \sqrt{3} \times 6 \sqrt{3}) \mathrm{R} 30^{\circ}$ periodicity [4] was grown in the same furnace at $1425^{\circ} \mathrm{C}$ for five minutes. For H-QFMLG hydrogen was intercalated between the buffer layer and the silicon carbide surface by annealing in an ultra-pure hydrogen atmosphere of 970 mbar pressure at $540^{\circ} \mathrm{C}$ for 75 minutes $[5,6]$. For O-QFMLG the buffer layer was annealed in water vapor[7] $\left(500^{\circ} \mathrm{C}, 30\right.$ minutes) which leads to the formation of a thin oxide layer between the $\mathrm{SiC}$ substrate and graphene. Quasi-freestanding bilayer graphene (H-QFBLG) on H-terminated $\mathrm{SiC}(0001)$ was prepared by annealing MLG in ultra-pure hydrogen [5] (860 ${ }^{\circ} \mathrm{C}, 75$ minutes).

All samples were characterized by x-ray photoelectron spectroscopy using monochromatized $\mathrm{AlK}_{\alpha}$ radiation in order to determine the layer thickness and to confirm successful intercalation. The homogeneity of all samples is biased by the high temperature growth step before intercalation [7]. The growth of a buffer layer is typically achieved with a very low level of monolayer impurities, as confirmed by scanning Raman spectroscopy. However our MLG samples typically show a small percentage of bilayer and buffer layer regions.

After transfer to the HREELS spectrometer, the samples were annealed at $350{ }^{\circ} \mathrm{C}$ in order to remove contaminations. The MLG, O-QFMLG and H-QFBLG samples were measured within a week after production. H-QFMLG samples were measured fresh as well as after prolonged exposure to ambient conditions, with no systematic difference in the HREELS spectra. However, the measured charge carrier mobilities do change from sample to sample as shown in the statistics published by Speck et al. [6].

\section{DIELECTRIC THEORY}

In the specular scattering geometry the probability for an electron to suffer an energy loss $\omega$ at a momentum transfer of $\vec{q}_{\|}$is given by $[8]$

$$
P\left(\vec{q}_{\|}, \omega\right)=\frac{e^{2}}{\pi^{2} \hbar q_{\|}}\left(\frac{2 q_{\|} v_{\perp}}{q_{\|}^{2} v_{\perp}^{2}+\left(\vec{q}_{\|} \cdot \vec{v}_{\|}-\omega\right)^{2}}\right)^{2} \times \Im\left(\frac{-1}{1+\bar{\epsilon}\left(\vec{q}_{\|}, \omega\right)}\right),
$$

where $\bar{\epsilon}\left(\vec{q}_{\|}, \omega\right)$ is the dielectric function and $v_{\perp}$ and $v_{\|}$are the components of the velocity of the electron perpendicular and parallel to the surface. The latter two quantities depend on the primary beam energy and the angle of incidence. Apart from the scattering geometry and the primary beam energy, it is therefore only necessary to calculate the surface loss function $\Im\left(\frac{-1}{1+\bar{\epsilon}(\vec{q} \|, \omega)}\right)$. To calculate the effective dielectric function $\bar{\epsilon}\left(\vec{q}_{\|}, \omega\right)$ we use the ansatz of a layered material, where every layer $i$ contributes phonon and plasmon excitations [8]:

$$
\epsilon^{i}(\omega)=\epsilon_{\infty}^{i}+\sum_{j} \frac{\Delta \epsilon^{i j} \omega_{p h}^{i j}{ }^{2}}{\omega_{p h}^{i j}-\omega^{2}-i \gamma^{i j} \omega}-\sum_{k} \frac{\omega_{p l}^{i k^{2}}}{\omega^{2}+i \Gamma^{i k} \omega} .
$$


Here, $\omega$ and $\gamma$ denote the phonon and plasmon energy and damping, respectively and $\Delta \epsilon$ is the excitation strength of each phonon contribution. The sums run over all possible phonon $\left(\omega_{p h}^{i j}\right)$ and plasmon $\left(\omega_{p l}^{i k}\right)$ modes of the layer $i$. The effective dielectric function of all layers is then calculated from the continued fraction

$$
\bar{\epsilon}=a_{1}-\frac{b_{1}^{2}}{a_{1}+a_{2}-\frac{b_{2}^{2}}{a_{2}+a_{3}-\frac{b_{3}^{2}}{a_{3}+\ldots}}} .
$$

The coefficients are $a_{i}=\epsilon_{i}(\omega) / \tanh \left(q_{\|} d_{i}\right)$ and $b_{i}=\epsilon_{i}(\omega) / \sinh \left(q_{\|} d_{i}\right)$, where $d_{i}$ is the thickness for each layer. Figures S1 and S2 show simulations of the HREELS spectrum for the best fit as well as for different sets of parameters, respectively.

[1] N. Sieber, T. Seyller, R. Graupner, L. Ley, R. Mikalo, P.Hoffmann, D. Batchelor, and D. Schmeißer, Mater. Sci. Forum 389-393, 717 (2002).

[2] K. V. Emtsev, A. Bostwick, K. Horn, J. Jobst, G. L. Kellogg, L. Ley, J. L. McChesney, T. Ohta, S. A. Reshanov, J. Röhrl, E. Rotenberg, A. K. Schmid, D. Waldmann, H. B. Weber, and T. Seyller, Nat. Mater. 8, 203 (2009).

[3] M. Ostler, F. Speck, M. Gick, and T. Seyller, Phys. Stat. Sol. B 247, 2924 (2010).

[4] K. V. Emtsev, F. Speck, T. Seyller, L. Ley, and J. D. Riley, Phys. Rev. B 77, 155303 (2008).

[5] F. Speck, M. Ostler, J. Röhrl, J. Jobst, D. Waldmann, M. Hundhausen, L. Ley, H. B. Weber, and T. Seyller, Mater. Sci. Forum 645-648, 629 (2010)

[6] F. Speck, J. Jobst, F. Fromm, M. Ostler, D. Waldmann, M. Hundhausen, H. B. Weber, and T. Seyller, Appl. Phys. Lett. 99, $122106(2011)$

[7] M. Ostler, F. Fromm, R. J. Koch, P. Wehrfritz, F. Speck, H. Vita, S. Böttcher, K. Horn, and T. Seyller, Carbon 70, 258 (2014).

[8] P. Lambin, J.-P. P. Vigneron, and A. A. Lucas, Comput. Phys. Commun. 60, 351 (1990). 


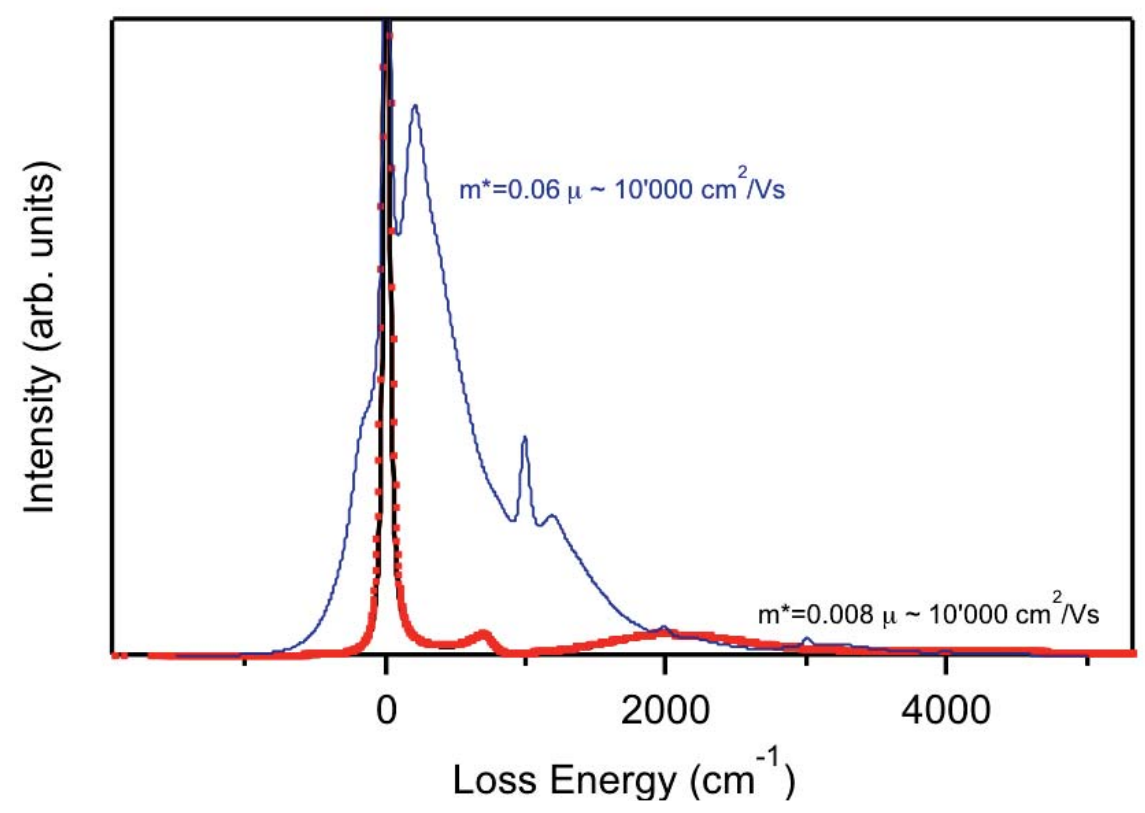

FIG. S 1. Full spectrum HREELS simulation using the best possible fitting parameters (black) and with $m^{*}=0.06$ (blue). The measurement is shown as red dots.

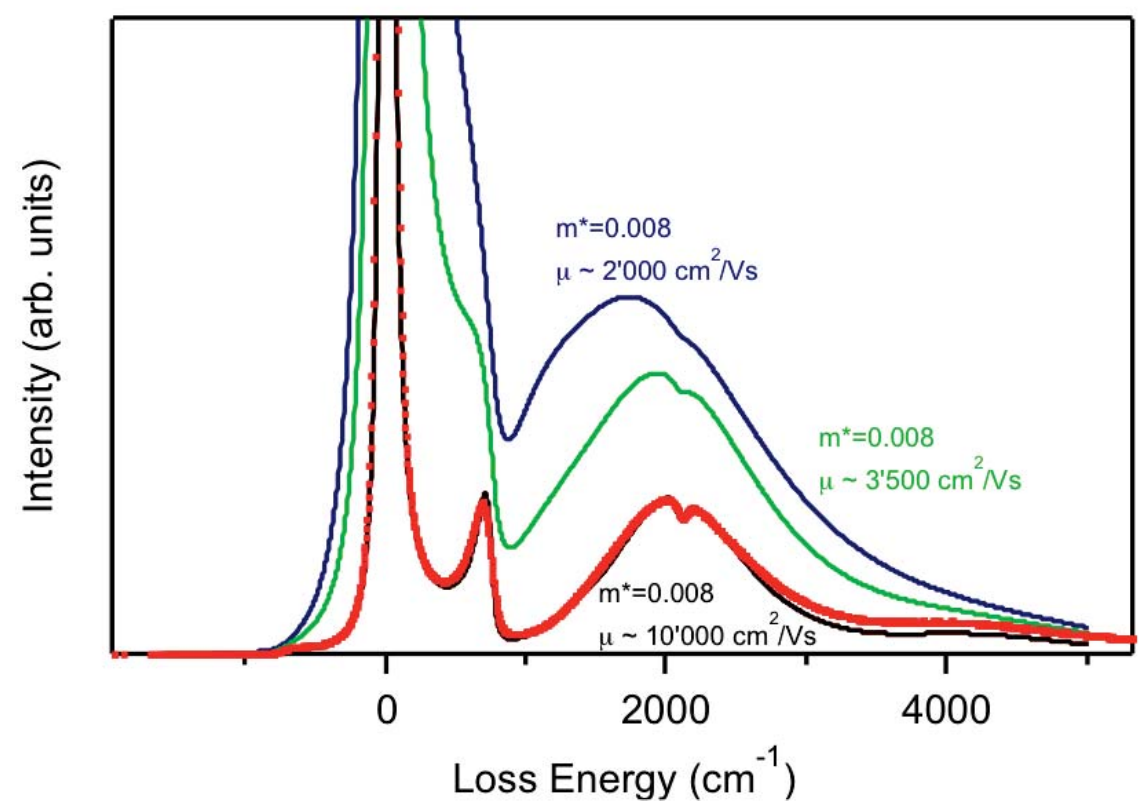

FIG. S 2. Full spectrum HREELS simulation using the best possible fitting parameters (black) and with $\mu \sim 2000 \mathrm{~cm}^{2} /$ Vs (blue) and $\mu \sim 3400 \mathrm{~cm}^{2} / \mathrm{Vs}$ (green). The measurement is shown as red dots. 